\title{
Integrative analysis of Geobacter spp. and sulfate-reducing bacteria during uranium bioremediation
}

\author{
M. Barlett ${ }^{1, *}$, K. Zhuang ${ }^{2, *}$, R. Mahadevan ${ }^{2}$, and D. Lovley ${ }^{1}$ \\ ${ }^{1}$ Department of Microbiology, Morrill Science Center IV North, University of Massachusetts, 639 North Pleasant St., \\ Amherst, MA, 01003-9298, USA \\ ${ }^{2}$ Department of Chemical Engineering and Applied Chemistry, University of Toronto, 200 College St. Rm 326, Toronto, \\ ON, M5S 3E5, Canada \\ *These authors contributed equally to this work.
}

Correspondence to: D. Lovley (dlovley@ microbio.umass.edu)

Received: 29 October 2011 - Published in Biogeosciences Discuss.: 24 November 2011

Revised: 18 February 2012 - Accepted: 28 February 2012 - Published: 16 March 2012

\begin{abstract}
Enhancing microbial U(VI) reduction with the addition of organic electron donors is a promising strategy for immobilizing uranium in contaminated groundwaters, but has yet to be optimized because of a poor understanding of the factors controlling the growth of various microbial communities during bioremediation. In previous field trials in which acetate was added to the subsurface, there were two distinct phases: an initial phase in which acetate-oxidizing, U(VI)-reducing Geobacter predominated and $\mathrm{U}(\mathrm{VI})$ was effectively reduced and a second phase in which acetate-oxidizing sulfate reducing bacteria (SRB) predominated and $\mathrm{U}(\mathrm{VI})$ reduction was poor. The interaction of Geobacter and SRB was investigated both in sediment incubations that mimicked in situ bioremediation and with in silico metabolic modeling. In sediment incubations, Geobacter grew quickly but then declined in numbers as the microbially reducible Fe(III) was depleted whereas the SRB grow more slowly and reached dominance after 30-40 days. Modeling predicted a similar outcome. Additional modeling in which the relative initial percentages of the Geobacter and SRB were varied indicated that there was little to no competitive interaction between Geobacter and SRB when acetate was abundant. Further simulations suggested that the addition of $\mathrm{Fe}$ (III) would revive the Geobacter, but have little to no effect on the SRB. This result was confirmed experimentally. The results demonstrate that it is possible to predict the impact of amendments on important components of the subsurface microbial community during groundwater bioremediation. The finding that $\mathrm{Fe}(\mathrm{III})$ availability, rather than competition with SRB, is the key factor limiting the activity
\end{abstract}

of Geobacter during in situ uranium bioremediation will aid in the design of improved uranium bioremediation strategies.

\section{Introduction}

Stimulating microbial reduction of soluble U(VI) to less soluble U(IV) with the addition of organic electron donors has shown promise as a strategy for preventing the spread of uranium in contaminated groundwater (Lovley, 2003; Wall and Krumholz, 2006; Williams et al., 2011). However, the added electron donors can also promote the activities of other microbial species, possibly hampering the effectiveness of bioremediation. For example, the addition of acetate to sulfate-rich groundwater at a uranium bioremediation study site in Rifle, $\mathrm{CO}$, produced an initial $\mathrm{Fe}(\mathrm{III})$ and $\mathrm{U}(\mathrm{VI})$ reducing phase, in which Geobacter species predominated, followed by a sulfate-reducing phase during which $\mathrm{Fe}$ (III) and $\mathrm{U}(\mathrm{VI})$ reduction ceased and acetate-oxidizing sulfate reducers related to Desulfobacter were more abundant (Anderson et al., 2003; Miletto et al., 2011). The transition from metal to sulfate reduction was accompanied by the increased abundance of sulfate-reducing bacteria (SRB), and took place between 40-60 days in both field and column studies (Anderson et al., 2003; Komlos et al., 2008; N'Guessan et al., 2008; Vrionis et al., 2005).

Previous studies on the interactions between Fe(III) reducers and sulfate reducers have focused on the competition for electron donors that takes place under steady-state conditions in sedimentary environments. In such environments, acetate 
and other more minor electron donors that support Fe(III) reduction and sulfate reduction are provided from the relatively slow hydrolysis and fermentation of complex organic matter (Lovley and Chapelle, 1995). Fe(III) reducers have a higher affinity for acetate and other electron donors than sulfate reducers, and, under steady-state conditions, can maintain the concentrations of these electron donors too low for sulfate reducers to metabolize (Lovley and Phillips, 1987; Chapelle and Lovley, 1992). If high concentrations of acetate or other electron donors are added to such sediments, then the competition for electron donors is relieved and $\mathrm{Fe}(\mathrm{III})$ reduction and sulfate reduction can proceed simultaneously (Lovley and Phillips, 1987).

Thus, it might be expected that the initial responses of Geobacter and Desulfobacter species to acetate amendments might be more comparable to each other, yet, the consistent observation is that Geobacter predominate in the early stages of acetate-driven in situ uranium bioremediation. An analysis of the competition between Geobacter and Rhodoferax species, which are also acetate-oxidizing Fe(III) reducers, demonstrated that although Rhodoferax could compete with Geobacter at the low rates of acetate production in unamended sediments, the addition of high concentrations of acetate favored Geobacter species which grow less efficiently but faster than Rhodoferax species (Zhuang et al., 2011). These results demonstrate that the selective pressures for competition are much different in environments with high concentrations of added electron donors versus environments in which electron donors are slowly provided via fermentation of complex organic matter.

In order to study the dynamic interactions between Geobacter and acetate-oxidizing SRB during uranium bioremediation, we have adopted an integrative approach that iteratively combined laboratory sediment experiments with the dynamic modeling of the Geobacter and SRB communities. Similar integrative approaches have been used to elucidate many important features of Geobacter physiology and ecology in recent years (Izallalen et al., 2008; Mahadevan et al., 2006, 2011; Segura et al., 2008; Sun et al., 2009; Zhuang et al., 2011; Mahadevan et al., 2011). The results of both experimental and modeling approaches suggest that Geobacter and SRB populations have little direct interaction and that the late appearance of SRB to added acetate can be attributed to a slower growth rate.

\section{Methods}

\subsection{Sediment incubations}

\subsubsection{Sediment incubation with acetate}

Sediments and groundwater were obtained from a uraniumcontaminated site at Old Rifle, CO, that has been described elsewhere (Anderson et al., 2003). Sediments and sterile, anaerobic groundwater were placed in glass bottles which were purged with a 95:5 $\mathrm{N}_{2}: \mathrm{CO}_{2}$ : gas mixture to achieve anaerobic conditions and then sealed with thick butyl rubber stoppers. Sodium acetate was added to provide an initial concentration of a concentration of $12 \mathrm{mM}$ and the sediments were incubated at $16^{\circ} \mathrm{C}$.

Sediments and groundwater were sampled under anaerobic conditions in an $\mathrm{N}_{2}$-filled glovebag. Acetate, sulfate, ferrous iron and total iron were determined as described below. A mixed sediment/groundwater slurry was immediately fixed for fluorescent in situ hybridization (FISH) in $4 \%$ paraformaldehyde $/ 0.5 \times$ phosphate buffered saline (PBS; $1 \times=7.6 \mathrm{~g} \mathrm{NaCl}, 1.9 \mathrm{~g} \quad \mathrm{Na}_{2} \mathrm{HPO}_{4} \cdot 7 \mathrm{H}_{2} \mathrm{O}$, $0.7 \mathrm{~g} \mathrm{NaH}_{2} \mathrm{PO}_{4} \cdot 2 \mathrm{H}_{2} \mathrm{O}$ per 1) with $0.1 \%$ sodium pyrophosphate and $0.15 \%$ Triton-X (Sigma) and stored at $4{ }^{\circ} \mathrm{C}$. This solution was vortexed for five seconds and allowed to settle briefly to remove the majority of sediment particles before being added to $0.2 \mu \mathrm{m}$ white polycarbonate filters (GTTP; Millipore, Billerica, MA) and washed with $1 \%$ Nonidet (Sigma) solution. Cells were hybridized for three hours as described previously (Lemke et al., 1997; Pernthaler et al., 2001) with a formamide concentration of $35 \%(v / v)$. The slides were embedded in Vectashield (Vector Laboratories, Burlingame, CA) and observed with a Nikon epifluorescence microscope. Ten to twenty fields of view for each sample were enumerated.

The number of Geobacter was determined by the number of cells that hybridized the probes GEO3A, GEO3B, and GEO3C (Richter et al., 2007). The number of sulfate reducing bacteria was inferred by determining the number of cells that hybridized the probes SRB385 and SRB385Db (Amann et al., 1990) and subtracting the number of Geobacter. This was done because the SRB385 and SRB385Db probes include the majority of SRB and some other deltaproteobacteria including the Geobacter (Rabus et al., 1996). The probe NON338 was used to account for autofluorescence (Wallner et al., 1993).

\subsubsection{Fe(III) oxide addition experiment}

After an initial experiment was run as above, bottles were incubated for over 2 months to fully reduce the bioavailable $\mathrm{Fe}(\mathrm{III})$ and sulfate in the sediments and groundwater. Once this initial reduction was complete, the groundwater was replaced with fresh groundwater containing natural concentrations of sulfate at about $8-10 \mathrm{mM}$ and additional acetate at a concentration of $2 \mathrm{mM}$. The bottles were incubated at $16^{\circ} \mathrm{C}$ for 17 days. At this point, they were further amended with acetate $(5 \mathrm{mM})$ in sulfate-containing groundwater and two of the three bottles were amended with poorly-crystalline $\mathrm{Fe}(\mathrm{III})$ oxide at a concentration of $50 \mathrm{mM}$. Acetate in groundwater was added on Days 23 and 27 to maintain concentrations between $2-5 \mathrm{mM}$. The bottles were sampled every $2-3$ days through Day 32 and samples were processed as above for geochemistry and bacterial cell numbers. 


\subsection{Analytical methods}

Groundwater samples for acetate and sulfate analyses were filtered through $0.2 \mu \mathrm{m}$ SFCA filters (Corning Inc.; Corning, NY) and measured on a Dionex ICS-1000 (Sunnyvale, $\mathrm{CA}$ ). Fe(II) and Fe(III) in the water and sediments were determined with the ferrozine method as previously described (Lovley and Phillips, 1987) after extraction in $0.5 \mathrm{~N} \mathrm{HCl}$ for $24 \mathrm{~h}$.

\subsection{Dynamic metabolic modeling of Geobacter and SRB}

A dynamic community metabolic model containing attached Geobacter, planktonic Geobacter, and SRB was used to study the metabolic interactions between Geobacter and SRB. Attached Geobacter were assumed to be the sole reducer of Fe(III) and planktonic Geobacter is assumed to be the sole reducer of uranium (Zhao et al., 2010). This particular model utilizes the genome-scale metabolic model of Geobacter (Zhuang et al., 2011) and a pathway-scale metabolic model of SRB; this model has been described elsewhere and been shown to be capable of predicting the microbial activities during the 2002 field bioremediation experiments at the study site (Zhuang et al., 2012).

\subsubsection{Determination of model parameters}

Using the methods described in Sect. 2.3, the total concentration of iron in the sediment has been determined to be $7.45 \mathrm{mM}$; about $70 \%(5.2 \mathrm{mM})$ of the iron has been determined to be $\mathrm{Fe}$ (III) which can be reduced by Geobacter, the remaining $2.25 \mathrm{mM}$ are $\mathrm{Fe}$ (II) which is already in a reduced form and cannot be further reduced by Geobacter. Previous work has established that in the Rifle sediment, less than $3 \%$ of the Fe(III) are in the easy-to-use amorphous oxide form, the rest are in more hard-to-use forms including $\mathrm{Fe}(\mathrm{III})$ silicate, Al-geothite, and magnetite (Komlos et al., 2008). Based on these experimental evidences, we have calculated the initial concentrations of Fe(II), Easy-to-Use Fe(III), and Hardto-Use Fe(III) to be $2.25 \mathrm{mM}, 0.16 \mathrm{mM}$, and $5.1 \mathrm{mM}$, respectively. In our model, Easy-to-Use and Hard-to-Use Fe(III) are treated as two different metabolites, whose dynamics are described separately.

The genome-scale model of Geobacter sulfurreducens has been updated to include separate pathways for the uptakes of Easy-to-Use Fe(III) and Hard-to-Use Fe(III). The uptake kinetics of acetate and Easy-to-Use Fe(III) for Geobacter have been described in a previous model (Zhuang et al., 2011); however, this model did not include the Hard-to-Use Fe(III) pathway. The saturation constant in the $\mathrm{Fe}$ (III) utilization kinetics for the easy-to-use $\mathrm{Fe}(\mathrm{III})$ have been previously published to be $1 \mathrm{mM}$ (Zhuang et al., 2011); here, we identified the $V_{\max }$ and $K_{\mathrm{S}}$ for hard-to-use $\mathrm{Fe}(\mathrm{III})$ using the $\mathrm{Fe}$ (II) data from the 2002 Rifle experiment (Supplement, Fig. S1) as well as the $\mathrm{Fe}(\mathrm{II})$ data from this work (Fig. 2f) (Supple-
A

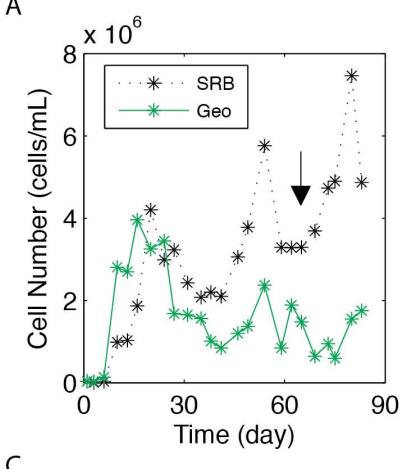

B

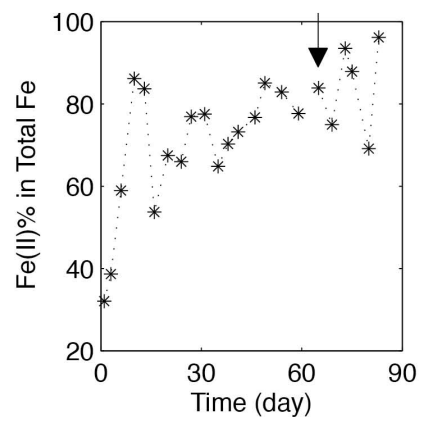

D
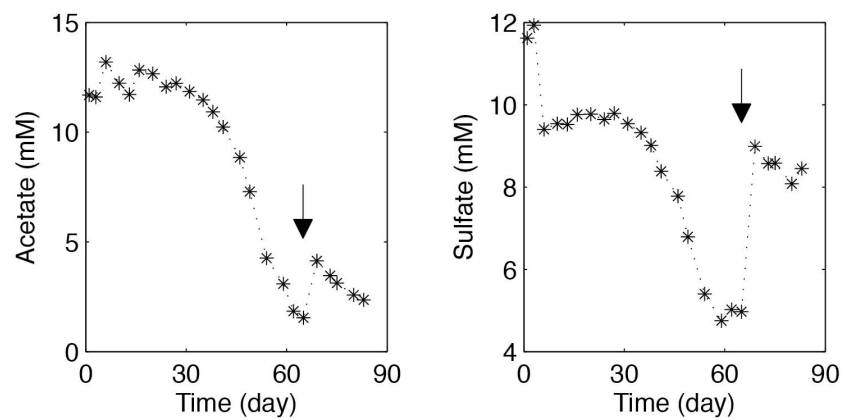

Fig. 1. Microbiological and geochemical impact of adding acetate to subsurface sediments. (A) The number of cells of Geobacter and sulfate-reducing bacteria (SRB) as determined by fluorescent in situ hybridization of probes $\mathrm{GeoA} / \mathrm{GeoB} / \mathrm{GeoC}$ and SRB385/SRB385Db, respectively; (B) Fe(III) reduction as indicated by an increase in the proportion of $\mathrm{Fe}$ (II) of the total acidextractable iron in the sediment; (C, D) The concentration of acetate (C) and sulfate (D) in the groundwater; The arrow denotes an addition of sterile groundwater to the batches that aimed to increase both acetate and sulfate by $5 \mathrm{mM}$ each; Data points are each an average of 5 separate batches.

ment, Fig. S1). The $V_{\max }$ for easy-to-use Fe(III) was set to $568 \mathrm{mmol} \mathrm{gdw}^{-1} \mathrm{~h}^{-1}$ as previously described (Zhuang et al., 2011). The $V_{\max }$ for hard-to-use Fe(III) was estimated to be $30 \mathrm{mmol} \mathrm{gdw}^{-1} \mathrm{~h}^{-1}$ using Fe(II) data.

Kinetic parameters for Desulfobacter postgatei (Ingvorsen et al., 1984), which is closely related to the majority of SRB that increase in abundance during the sulfate reduction phase (Miletto et al., 2011), were employed in the SRB modeling with the exception that the published kinetics in our experimental data (Fig. 1) show that the Rifle SRB's affinity for sulfate is much lower than that of the laboratory Desulfobacter strains during the batch sediment incubation. A $K_{\mathrm{s}}$ value of $13 \mathrm{mM}$ was estimated using the experimental sulfate data (Fig. 2e).

A death rate of $0.0011 \mathrm{~h}^{-1}$ was chosen for both organisms, which is based on the value of two previous models of sulfate and iron reducers (Bethke et al., 2008; Moosa, 2002). This value can describe the decay of Geobacter (Fig. 2). 
A

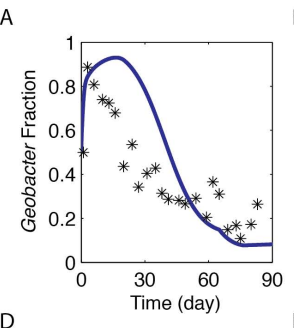

D
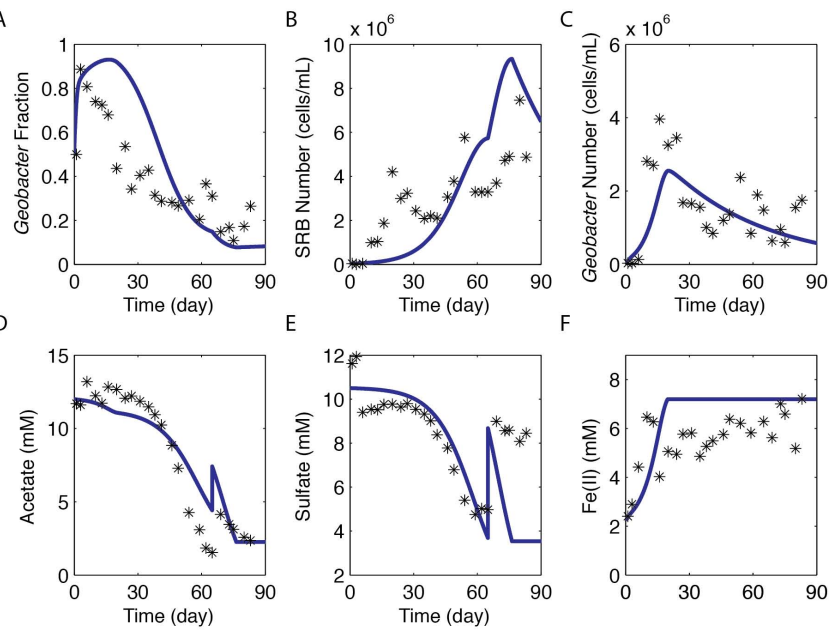

Fig. 2. In silico predictions of trends in the fraction of Geobacter in the community (A), number of SRB (B), number of Geobacter $(\mathbf{C})$, acetate concentration (D), sulfate concentration (E), and Fe(II) concentration $(\mathbf{F})$. Lines are the in silico predictions; * are the experimental values. The experimental $\mathrm{Fe}$ (II) values are calculated by multiplying the total iron concentration of $7.5 \mathrm{mM}$ with the experimentally measured $\mathrm{Fe}$ (II) percentage in total Fe(II) from Fig. 1.

\subsubsection{Simulations of batch incubation experiments}

To model the laboratory sediment incubation experiment with acetate amendment only, the initial concentrations of acetate and sulfate were set to $12 \mathrm{mM}$ and $10.5 \mathrm{mM}$, and the initial cell concentrations of both organisms to $3.4 \times$ $10^{4}$ cells $\mathrm{ml}^{-1}$. These values are representative of the measured experimental conditions during sediment incubation. Additions of acetate and sulfate were made in silico on day 65 to reflect the additions in the laboratory experiment.

Ideal batch reactor models were used to simulate conditions with varying starting proportions of Geobacter and SRB that were impossible to replicate in the lab. The initial cell number for all organisms was assumed to be $10^{4}$ cells $\mathrm{ml}^{-1}$, which is similar to the cell number measured in the batch sediment incubation experiment. These cells were then divided between Geobacter and SRB for varying percentage starts including $100 \%, 90 \%$, and $50 \%$ of each group. The SRB were determined to have twice as much mass/cell as the Geobacter as calculated from the cellular dimensions found in the FISH pictures of each group.

The model used to simulate Fe(III) amendment was initialized with the same conditions as in the acetate-amended batch incubation simulation except no additional acetate and sulfate was added later in the experiment. Two simulations were performed: $5 \mathrm{mM}$ of Easy-to-Use Fe(III) was added on day 45 in one simulation and no $\mathrm{Fe}$ (III) was added in the other simulation.

\section{Results and discussion}

\subsection{Sediment incubation with acetate and its simulation}

The interaction of Geobacter species and SRB was evaluated in sediment incubations that simulated conditions in the field experiments, but provided the opportunity to quantify the number of cells in each population over time. With the addition of acetate there was a rapid growth of Geobacter species (Fig. 1), as previously observed in field studies (Anderson et al., 2003; Vrionis et al., 2005; Holmes et al., 2012). Adding acetate also stimulated the growth of SRB, but they grew more slowly (Fig. 1). After day 24, the number of Geobacter species declined, coincident with reduction of ca. $80 \%$ of the Fe(III) in the sediment (Fig. 1). However, the SRB continued to increase in number and became predominant (Fig. 1). Addition of more acetate and sulfate as they became depleted stimulated additional growth of SRB, but not Geobacter species. This pattern of succession from Geobacter to SRB has previously been observed in field and column studies (Anderson et al., 2003; Komlos et al., 2008; Milleto et al., 2011) and the timing of the transition to SRB predominance in the batch studies reported here is similar to that seen in those previous studies.

These results demonstrated concurrent growth of both Geobacter species and SRB following the addition of acetate, but it was not possible to elucidate from these studies whether the metabolism of the two metabolic groups had an influence on each other. Therefore, the potential for such interactions was further investigated with a dynamic community metabolic model of Geobacter and SRB to investigate the metabolic interactions between these two organisms.

First, the sediment incubation study was modeled in order to check the model validity. The model was able to predict the growth of Geobacter species and SRB, and the uptake of acetate and sulfate, the reduction of $\mathrm{Fe}$ (III), as well as the evolution of the fraction of cells that are Geobacter species (Fig. 2). Like the sediment incubation experiment, the simulation predicted that a batch addition of acetate would lead to the initial dominance of Geobacter species and the latter overtaking of Geobacter by the SRB (Fig. 2). The higher biomass yield and the maximum acetate uptake rate of Geobacter resulted in Geobacter growing faster than SRB when $\mathrm{Fe}(\mathrm{III})$ was abundant. However, the predicted growth rate of Geobacter decreased drastically once the Easy-to-Use $\mathrm{Fe}(\mathrm{III})$ was exhausted; its growth eventually stopped after the exhaustion of all available Fe(III). On the other hand, SRB grew slowly but steadily, overtaking Geobacter species between day 30 and 40. The growth rate of SRB did not vary before and after the exhaustion of Fe(III), suggesting that the microbial reduction of $\mathrm{Fe}(\mathrm{III})$ had little effect on the growth of SRB. Thus, despite its relative simplicity, the model was able to predict the shifts in community composition as well as the trends of metabolite utilization observed in the sediment incubations. 
It is important to note that while the model was able to predict the general trends, it could not capture finer details. For example, three peaks were observed in the Geobacter and SRB numbers, which could not be predicted individually. It is possible that the peaks were caused by sampling errors due to the highly heterogeneous nature of the sediment. Another possibility is that different strains of Geobacter and SRB, which are known to exist within the Rifle sediment, grew at different rates; since we only modeled one species of Geobacter and SRB respectively, the different peaks could not be predicted. Lastly, the Rifle sediment contains several acetate-consuming species that we did not include in the model; although they are much less influential than Geobacter and SRB (Anderson et al., 2003; Vrionis et al., 2005), they can have a minor influence the community dynamics. Ultimately, these discrepancies between the model and the experimental data can help us improve our model as well as highlighting our knowledge gaps regarding the subsurface community.

\subsection{Simulations with varying initial microbial fractions}

A potentially important consideration in understanding the interactions between Geobacter species and SRB following the addition of acetate to groundwater is the composition of the microbial community prior to acetate amendment. For example, previous molecular analysis of the subsurface community prior to the addition of acetate found that about $5 \%$ of the Rifle microbial community were Geobacter species, whereas sequences that could be attributed to acetate-using SRB were not detectable (Holmes et al., 2005; Miletto et al., 2011; Mouser et al., 2009) possibly giving Geobacter an initial numerical advantage over the SRB. To mimic the natural variations in the abundance of Geobacter and SRB prior to the addition of acetate, simulations were run with a community that was composed of $0 \%, 10 \%, 50 \%, 90 \%$, and $100 \%$ Geobacter at the onset. The simulations demonstrated that the abundance of Geobacter and SRB prior to acetate addition had very little influence on the community dynamics after the acetate addition. In all the simulations containing Geobacter, they quickly became the dominant species after acetate addition, increasing their numbers to about $2 \times 10^{6}$ cells $\mathrm{ml}^{-1}$ before the depletion of bioavailable Fe(III) (Fig. 3, Row 1). SRB grew at a much slower rate than Geobacter species, but SRB growth continued for a longer time because of the abundance of sulfate in the system. In all simulations containing SRB, they overtook Geobacter in abundance at about day 23 and increased their numbers to

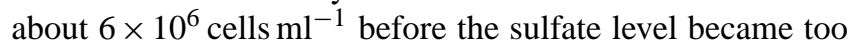
low to maintain growth, (Fig. 3, Row 1). Acetate, Fe(III), and sulfate were consumed at expected times and quantities comparable to the growth of Geobacter and SRB.

Strikingly, the dynamic features of $\mathrm{Fe}(\mathrm{III})$ and sulfate reduction in all the simulations containing Geobacter and SRB respectively are practically identical. It appears that the dy-
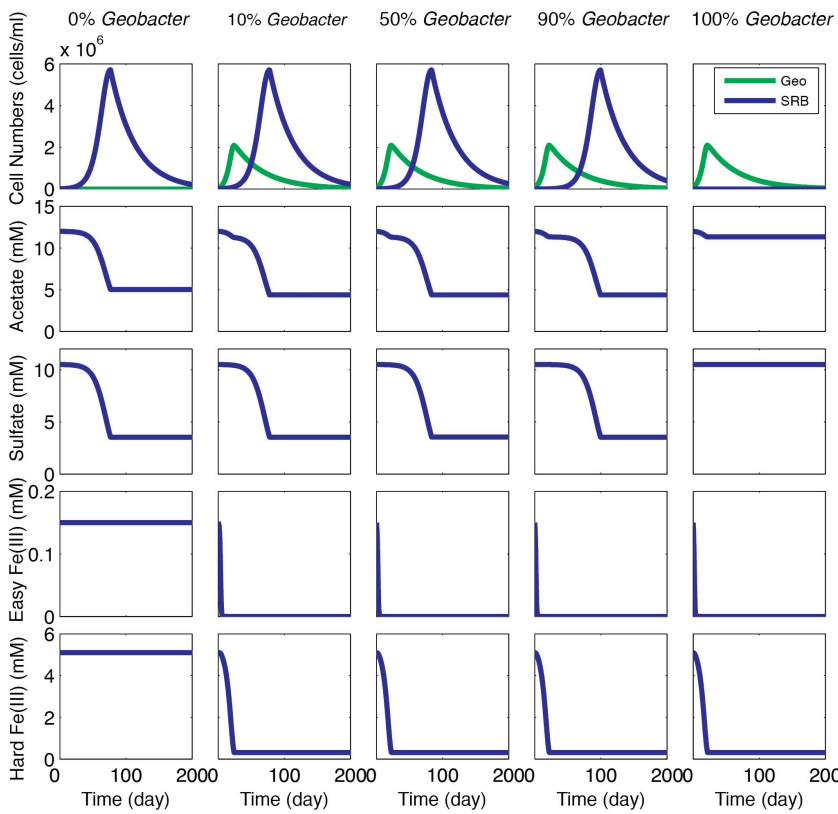

Fig. 3. Ideal batch reactor model simulations with varying initial ratio of Geobacter and SRB. In the cell number row, green line indicates Geobacter, blue line indicates SRB.

namics of the simulations containing both organisms is equal to the dynamics of the simulation containing no Geobacter plus the dynamics of the simulation containing only Geobacter. The difference in the timing of the onset of sulfate reduction between the simulations with $90 \%$ and $10 \% \mathrm{SRB}$ is only about a day. Even with no Geobacter present, it still took more than 30 days for the sulfate reduction to become apparent (Fig. 3, Column 2-5). Acetate never became limiting for any of the simulations, indicating that SRB were never competitively excluded under these conditions. These simulations clearly demonstrate that the metabolic interactions between Geobacter and SRB are minimal following a batch addition of a high dosage of acetate. A similar lack of interaction is expected in the field where the acetate concentration is maintained at a high level due to the continuous addition of acetate (Williams et al., 2011).

The same conclusions can be drawn from simulations initiated with 100 times less initial biomass and simulations initiated with twice as much Fe(III) (Figs. S2 and S3 in Supplement). However, in the low initial biomass simulation, the onset of sulfate reduction and the time at which the SRB overtook the Geobacter was significantly delayed (Fig. S2 in Supplement). This delay occurred because it took additional time for SRB to accumulate a sufficiently high biomass to become a major contributor of the community metabolism. Given that the timing of the onset of sulfate reduction is the same in the cases with Geobacter and in the case without Geobacter, it is clear that the lateness of the onset of sulfate reduction is primarily due to the slow growth rate of SRB and 
A

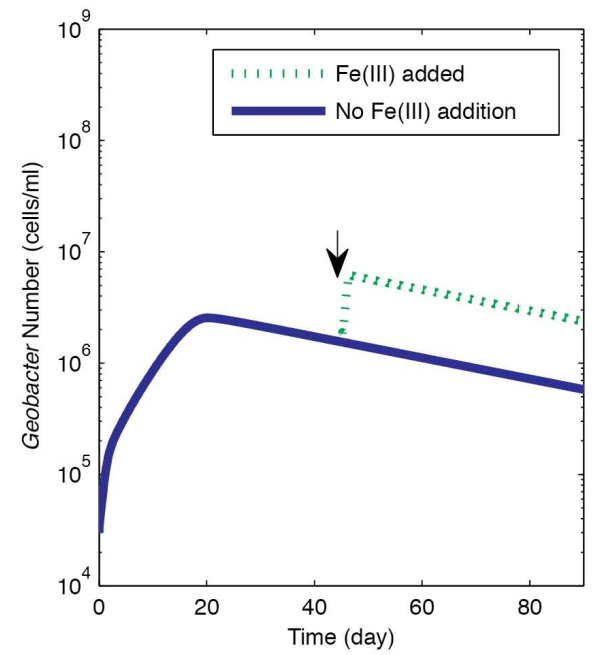

B

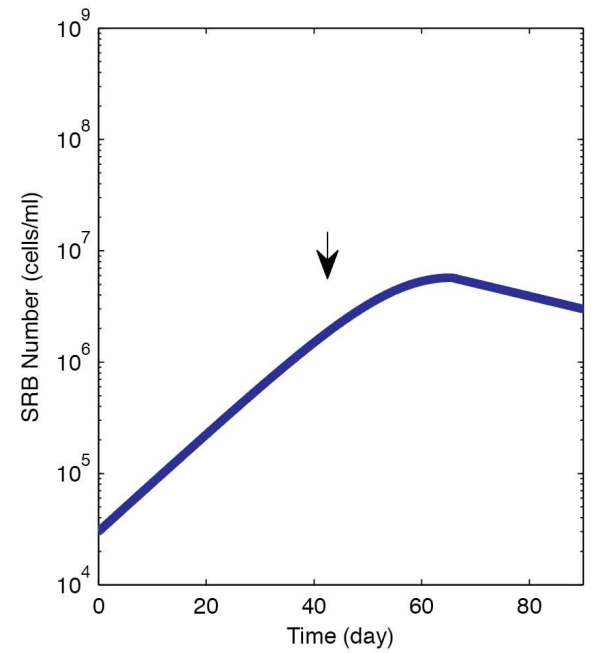

Fig. 4. Predicted impact of adding Fe(III) on the number of Geobacter (A) and SRB (B). The different line style and markers distinguish the control simulation where no Fe(III) was added from the simulation where Fe(III) was added. The black arrows indicates the day Fe(III) was added.
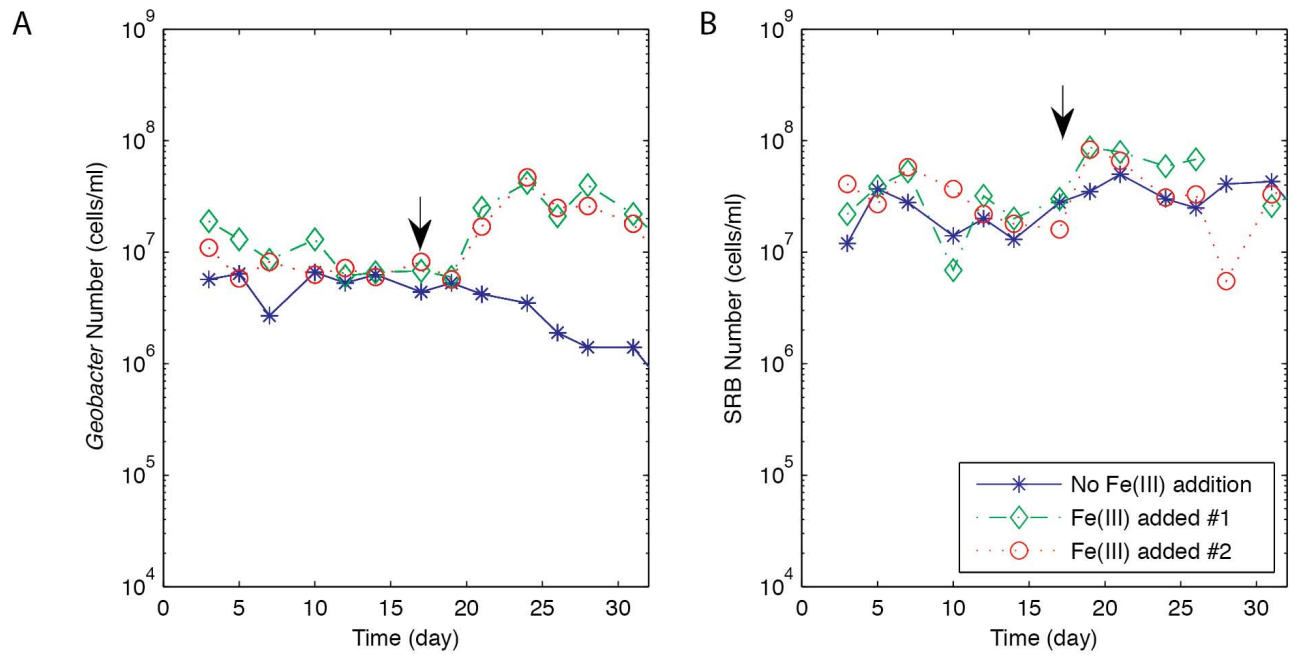

Fig. 5. Observed Impact of Fe(III) oxide additions on the number of Geobacter (A) and SRB (B). The green (diamond style) and red (circle style) lines indicates the data from two bottles in which Fe(III) was added. The blue (star style) line indicates the data from the bottle in which no Fe(III) was added. The black arrows indicates the time Fe(III) was added to both bottles \#1 and \#2.

is modulated by the initial abundance of SRB, and it is therefore unrelated to the presence of Geobacter. Furthermore, the fact that that the timing of the decrease of Geobacter activities is the same in the cases with or without SRB, it is clear that the growth of Geobacter is limited by Fe(III) availability alone, and is unrelated to the competition from SRB for acetate.

Thus, both the sediment incubation studies (Fig. 1) and the simulations (Figs. 2 and 3) suggest that Geobacter and SRB are not mutually exclusive during bioremediation. In fact, the results demonstrated that neither organism significantly inhibits the activity of its competitor as long as the common nutrient is abundantly available.

\subsection{Effects of adding $\mathrm{Fe}$ (III) oxide}

The apparent lack of interaction between Geobacter species and SRB suggested that the addition of more Fe(III) oxide to the system might prolong the growth and activity of Geobacter species. A simulation of $\mathrm{Fe}$ (III) oxide amendment predicted an increase in Geobacter in response to Fe(III) additions with no changes in SRB growth or sulfate reduction (Fig. 4). To experimentally test the model predictions, acetate and sulfate, and then acetate, sulfate, and Fe(III) oxide were added to sediments which were previously amended with acetate and incubated until $\mathrm{Fe}(\mathrm{III})$ and sulfate were depleted. Geobacter grew in response to the Fe(III) additions 
in the two bottles where $\mathrm{Fe}$ (III) was amended but they did not grow when no Fe(III) was added (Fig. 5a). SRB grew in response to the additions of acetate and sulfate and the addition of $\mathrm{Fe}$ (III) had little effect on their growth (Fig. 5b) and the reduction of sulfate (data not shown). Similarly, simulation showed that if $\mathrm{Fe}$ (III) is added to the sediment at the beginning of the experiment, additional Geobacter biomass is produced, leading to faster utilization of $\mathrm{Fe}(\mathrm{III})$ but having little effect on the utilization of sulfate (Supplement Fig. S4). These results were consistent with the model predictions, demonstrating that the addition of $\mathrm{Fe}(\mathrm{III})$ oxide can resuscitate the growth of Geobacter, and further suggesting that the activities of Geobacter and SRB are not mutually exclusive during acetate-rich phases of bioremediation.

\section{Concluding remarks}

The results demonstrate that it is possible to predictively model the interaction of growth of Geobacter and SRB when acetate is added to subsurface sediments to promote in situ uranium reduction. It is apparent from both the experimental and modeling approaches that the acetate-oxidizing Geobacter and SRB have little impact on each other as long as acetate is maintained in excess. The growth of Geobacter species is primarily controlled by the availability of $\mathrm{Fe}(\mathrm{III})$, and the initial predominance of Geobacter species following the addition of acetate can be attributed to the faster growth of Geobacter species. These studies provide the basis for the modeling-based design of in situ bioremediation approaches which will also have to consider additional complexities, such as rates of acetate delivery to the subsurface via injection wells, and geochemical reactions, such as the reduction of $\mathrm{Fe}(\mathrm{III})$ by sulfide, which removes $\mathrm{Fe}$ (III), but also generates $\mathrm{S}^{\circ}$, an alternative electron acceptor for Geobacter species. Through this approach, it is expected that the optimal strategies for the addition of acetate, $\mathrm{Fe}(\mathrm{III})$, and possibly other amendments will be identified.

\section{Supplementary material related to this article is available online at: http://www.biogeosciences.net/9/1033/2012/ bg-9-1033-2012-supplement.pdf.}

Acknowledgements. We acknowledge support by the Office of Science (BER), US Department of Energy, Awards No. DESC0004080, No. DE-SC0004814, and Cooperative Agreement No. DE-FC02-02ER63446. In addition, K. Z. and R. M. acknowledge support by the Office of Science (BER), US Department of Energy, Grant No. DE-FG02-07ER64367, Genome Canada as well as Natural Sciences and Engineering Research Council of Canada, Ontario Ministry of Research and Innovation, Canada Foundation for Innovation and University of Toronto Open Fellowship.

Edited by: K. Küsel

\section{References}

Amann, R. I., Binder, B. J., Olson, R. J., Chisholm, S. W., Devereux, R., and Stahl, D. A.: Combination of 16S rRNA-targeted oligonucleotide probes with flow cytometry for analyzing mixed microbial populations., Appl. Environ. Microbiol., 56, 19191925, 1990.

Anderson, R. T., Vrionis, H. A., Ortiz-Bernad, I., Resch, C. T., Long, P. E., Dayvault, R., Karp, K., Marutzky, S., Metzler, D. R., Peacock, A., White, D. C., Lowe, M. and Lovley, D. R.: Stimulating the In Situ Activity of Geobacter Species to Remove Uranium from the Groundwater of a Uranium-Contaminated Aquifer, Appl. Environ. Microbiol., 69, 5884-5891, 2003.

Bethke, C. M., Ding, D., Jin, Q., and Sanford, R. A.: Origin of microbiological zoning in groundwater flows, Geology, 36, 739$742,2008$.

Chapelle, F.: Competitive exclusion of sulfate reduction by $\mathrm{Fe}(111)$-reducing bacteria: A mechanism for producing discrete zones of high-iron ground water, Ground Water, 30, 29-36, doi:10.1111/j.1745-6584.1992.tb00808.x, 1992.

Elferink, S., Luppens, S., Marcelis, C., and Stams, A.: Kinetics of acetate oxidation by two sulfate reducers isolated from anaerobic granular sludge, Appl. Environ. Microbiol., 64, 2301-2303, 1998.

Holmes, D. E., Nevin, K. P., O’Neil, R. A., Ward, J. E., Adams, L. A., Woodard, T. L., Vrionis, H. A., and Lovley, D. R.: Potential for quantifying expression of the Geobacteraceae citrate synthase gene to assess the activity of Geobacteraceae in the subsurface and on current-harvesting electrodes, Appl. Environ. Microbiol., 71, 6870-6877, 2005.

Holmes, D. E., Barlett, M. A., Chavan, M. A., Smith, J. A., Giloteaux, L., Risso, C., Williams, K. H., Wilkins, M., Long, P. E., and Lovley, D. R.: Molecular analysis of the growth rate of subsurface Geobacter species during in situ uranium bioremediation, Appl. Environ. Microbiol., submitted, 2012.

Ingvorsen, K., Zehnder, A. J. B., and Jorgensen, B. B.: Kinetics of Sulfate and Acetate Uptake by Desulfobacter postgatei, Appl. Environ. Microbiol., 47, 403-408, 1984.

Izallalen, M., Mahadevan, R., Burgard, A., Postier, B., Didonato, R., Sun, J., Schilling, C. H., and Lovley, D. R.: Geobacter sulfurreducens strain engineered for increased rates of respiration, Metab. Eng., 10, 267-275, 2008.

Komlos, J., Moon, H. S., and Jaffé, P. R.: Effect of Sulfate on the Simultaneous Bioreduction of Iron and Uranium, J. Environ. Qual., 37, 2058-2062, 2008.

Lemke, M. J., McNamara, C. J., and Leff, L. G.: Comparison of methods for the concentration of bacterioplankton for in situ hybridization, J. Microbiol. Meth., 29, 23-29, 1997.

Lovley, D. R.: Cleaning up with genomics: applying molecular biology to bioremediation, Nat. Rev. Microbiol., 1, 35-44, 2003.

Lovley, D. R. and Phillips, E. J.: Competitive mechanisms for inhibition of sulfate reduction and methane production in the zone of ferric iron reduction in sediments., Appl. Environ. Microbiol., 53, 2636-2641, 1987.

Mahadevan, R., Bond, D. R., Butler, J. E., Esteve-Nuñez, A., Coppi, M. V., Palsson, B. O., Schilling, C. H., and Lovley, D. R.: Characterization of metabolism in the $\mathrm{Fe}(\mathrm{III})$-reducing organism Geobacter sulfurreducens by constraint-based modeling, Appl. Environ. Microbiol., 72, 1558-1568, 2006.

Mahadevan, R., Palsson, B. Ø., and Lovley, D. R.: In situ to in silico 
and back: elucidating the physiology and ecology of Geobacter spp. using genome-scale modelling., Nat. Rev. Microbiol., 9, 3950, 2011.

Miletto, M., Williams, K. H., N'Guessan, A. L., Lovley, and D. R.: Molecular analysis of the metabolic rates of discrete subsurface populations of sulfate reducers, Appl. Environ. Microbiol. 77, 6502-6509, 2011.

Moon, H. S., McGuinness, L., Kukkadapu, R. K., Peacock, A. D., Komlos, J., Kerkhof, L. J., Long, P. E., and Jaffe, P. R.: Microbial reduction of uranium under iron- and sulfate-reducing conditions: Effect of amended goethite on microbial community composition and dynamics, Water Res., 44, 4015-4028, 2010.

Moosa, S.: A kinetic study on anaerobic reduction of sulphate, Part I: Effect of sulphate concentration, Chem. Eng. Sci., 57, 27732780, 2002.

Mouser, P. J., N'Guessan, A. L., Holmes, D. E., Williams, K. H., Wilkins, M. J., Long, P. E., and Lovley, D. R.: Influence of heterogeneous ammonium availability on bacterial community structure and the expression of nitrogen fixation and ammonium transporter genes during in situ bioremediation of uraniumcontaminated groundwater, Environ. Sci. Technol., 43, 43864392, 2009.

N'Guessan, A. L., Vrionis, H. A., Resch, C. T., Long, P. E., and Lovley, D. R.: Sustained removal of uranium from contaminated groundwater following stimulation of dissimilatory metal reduction, Environ. Sci. Technol., 42, 2999-3004, 2008.

Pernthaler, J., Glockner, F., Schönhuber, W., and Amann, R.: Methods in Microbiology, Elsevier. 2001.

Rabus, R., Fukui, M., Wilkes, H., and Widdle, F.: Degradative capacities and 16S rRNA-targeted whole-cell hybridization of sulfate-reducing bacteria in an anaerobic enrichment culture utilizing alkylbenzenes from crude oil, Appl. Environ. Microbiol., 62, 3605-3613, 1996.

Reeburgh, W. S.: Rates of biogeochemical processes in anoxic sediments, Annu. Rev. Earth Pl. Sc., 11, 269-298, 1983.

Richter, H., Lanthier, M., Nevin, K. P., and Lovley, D. R.: Lack of Electricity Production by Pelobacter carbinolicus Indicates that the Capacity for Fe(III) Oxide Reduction Does Not Necessarily Confer Electron Transfer Ability to Fuel Cell Anodes, Appl. Environ. Microbiol., 73, 5347-5353, 2007.
Schönheit, P., Kristjansson, J. K., and Thauer, R. K.: Kinetic mechanism for the ability of sulfate reducers to out-compete methanogens for acetate, Arch. Microbiol., 132, 285-288, 1982.

Segura, D., Mahadevan, R., Juárez, K., and Lovley, D. R.: Computational and experimental analysis of redundancy in the central metabolism of Geobacter sulfurreducens., PLoS Comput. Biol., 4, e36, doi:10.1371/journal.pcbi.0040036, 2008.

Sun, J., Sayyar, B., Butler, J. E., Pharkya, P., Fahland, T. R., Famili, I., Schilling, C. H., Lovley, D. R., and Mahadevan, R.: Genomescale constraint-based modeling of Geobacter metallireducens, BMC Syst. Biol., 3, doi:10.1186/1752-0509-3-15, 2009.

Vrionis, H. A., Anderson, R. T., Ortiz-Bernad, I., O’Neill, K. R., Resch, C. T., Peacock, A. D., Dayvault, R., White, D. C., Long, P. E., and Lovley, D. R.: Microbiological and geochemical heterogeneity in an in situ uranium bioremediation field site, Appl. Environ. Microbiol., 71, 6308-6318, 2005.

Wall, J. D. and Krumholz, L. R.: Uranium reduction, Annu. Rev. Microbiol. 60, 149-166, 2006.

Wallner, G., Amann, R., and Beisker, W.: Optimizing fluorescent in situ hybridization with rRNA-targeted oligonucleotide probes for flow cytometric identification of microorganisms, Cytometry, 14, 136-143, 1993.

Williams, K. H., Long, P. E., Davis, J. A., Wilkins, M. J., N'Guessan, A. L., Steefel, C. I., Yang, L., Newcomer, D., Spane, F. A., Kerkhof, L. J., McGuinness, L., Dayvault, R., and Lovley, D. R.: Acetate Availability and its Influence on Sustainable Bioremediation of Uranium-Contaminated Groundwater, Geomicrobiol. J., 28, 519-539, 2011.

Zhao, J., Fang, Y., Scheibe, T. D., Lovley, D. R., and Mahadevan, R.: Modeling and sensitivity analysis of electron capacitance for Geobacter sedimentary environment, J. Contam. Hydrol., 112, 30-44, 2010.

Zhuang, K., Izallalen, M., Mouser, P., Richter, H., Risso, C., Mahadevan, R., and Lovley, D. R.: Genome-scale dynamic modeling of the competition between Rhodoferax and Geobacter in anoxic subsurface environments., ISME J., 5, 305-316, 2011.

Zhuang, K., Ma, E., and Mahadevan, R.: The Design of Long-term Effective Uranium Bioremediation Strategy using a Community Metabolic Model, Biotechnol. Bioeng., submitted, 2012. 